\title{
Evolutionstheorie in der Diskussion
}

\author{
Von Rolf Kühn
}

Die Berechtigung von psychosomatischer Medizin und therapiebezogener Psychologie wird, im Zuge des Zeitgeistes, oft an ihrer «Wissenschaftlichkeit» gemessen. Daß dieser Begriff, soweit er die moderne Biologie und Biochemie betrifft, weitgehend ideologischer Natur ist, erweist eine neuere Untersuchung von Dieter Wyss, worin er repräsentative Aussagen aus dem Bereich der Evolutions- und genetischen Informationstheorie untereinander konfrontiert:

Dieter Wyss, Vom zerstörten zum wiederentdeckten Leben. Kritik der modernen Biologie (Neue Wege der psychosomatischen Medizin, Bd.I), Vandenhoeck \& Ruprecht, Göttingen 1986, 260 Seiten. - Die Seitenzahlen im Text beziehen sich hierauf.

Ohne die experimentellen Einzelleistungen zu schmälern, kann nach einem solchen Vergleich nicht mehr darüber hinweggesehen werden, daß größte Widersprüche unter den Biologen selbst hinsichtlich ihrer theoretischen Grundkonzepte und deren Modellanwendungen bestehen.

Dies bliebe eine rein wissenschaftstheoretische Problematik, wenn nicht zugleich die «Ergebnisse» der gegenwärtigen Biologie zu Lehrsätzen erhoben würden, die einem positivistisch-materialistischen Bewußtsein im allgemeinen verpflichtet sind und einen populärwissenschaftlichen Neodarwinismus insbesondere erzeugen. Die weitere Gefahr hiervon ist, daß die Medizin und verwandte Wissenschaften zum bloßen «Ableger» (S.10) einer solchen wissenschaftlich verbrämten Weltanschauung werden und der kranke Mensch zunehmend einer ebenso ganzheitlich-komplexen wie differenziert-subjektbezogenen Wahrnehmungsweise entgleitet.

Die Frage nach der Entstehung von Erde und Leben als Prolegomena zu einem anthropologischen Gesamtverständnis zu stellen, heißt deshalb bereits von den evolutiven Bedingungen her den Blick zu schärfen für einen Prozeß, bei dem die Verstehenskategorien aus dem organismischen Gesamtgeschehen selbst heraus zu entfalten sind. 


\section{Zur Lebensentstehung}

Schon hier stößt man auf ein methodisches Grundproblem, insofern die These von der «chemischen Evolution» («Ur-» oder «Eiweißsuppe») auf Laborsimulierungen beruht, bei der notwendige Voraussetzungen gemacht werden, die erst zu beweisen wären, und zwar sowohl hinsichtlich des physikalischen Erdzustandes sowie bezüglich seiner (bio)chemischen Bestandteile (cf. S.29f.). Von besonderer Erforschungsaktualität bleiben hier deshalb der reale Zustand der Erdatmosphäre und Ozeane über «mehrere Milliarden Jahre» hinweg als auch die Herkunft der enzymatischen «Eigenschaften» der sich replizierenden biologischen Erstmoleküle.

$\mathrm{Da} ß$ die «richtigen» Moleküle sich zusammenfügen und «anpassend» ihre «Nahrung» suchen, setzt im Grunde den Begriff Leben schon voraus, worüber eine mechanistisch reduzierte Teleologie als «Teleonomie» nur schwerlich hinwegtäuschen kann. Die Inanspruchnahme der darwinistischen Vorstellung von «Selektion» auf der Ebene «lern-» und «informations»-verarbeitender Molekularprozesse kann zudem ebenso lamarckistisch interpretiert werden, was zeigt, daß die biologische «Systemtheorie» neueren Datums heterogenste Theorieelemente umfaßt. Sie sollen dazu dienen, den Darwinismus «chemisch» zu erhärten, indem ein solcher Sollwert des Lebendigen wie die «Erfahrungsweitergabe» als angeblich molekularfunktional identifiziert wird. Konsequenterweise müßte aber dann jedes Molekül Informationsträger sein, was keineswegs der Fall ist. Die Vorstellung eines «Hyperzyklus» - wie bei M.Eigen z. B. - besteht letztlich darin,

«die in allen Lebewesen vorhandene molekular-unauflösbare Abhängigkeit der DNA-, mRNA-, tRNA-Protein-Synthesen durch die Polymerasen u. a. Enzyme in ein zeitliches Nacheinander aufzulösen, erst die RNA entstehen zu lassen, dann die Enzyme, dann die DNA - nach der Entstehung der Polymerase - und sie endlich im Hyperzyklus zusammenzuschließen. Die in ihre 〈Elemente〉 zerlegte «Vererbungsmaschinerie) wird in ein zeitliches Nacheinander aufgeteilt, das dann wieder zu einem räumlich gegenseitig abhängigen katalytischen ¿Zyklus` zusammengefügt wird» (S.45).

Die «Metapher» einer solchen «Selbstorganisation» verstößt gegen den zweiten thermodynamischen Hauptsatz, denn die anaerob «lebenden» Biomoleküle müßten das biochemische Prinzip des Bindungsaustausches und damit der Entropie «übersprungen» haben, um Neg-Entropie und Information - d.h. «Leben» - entstehen zu lassen. Was jedoch - außer einem mathematisch präsentierten Finalismus von «fitness» und «quasi-species»- 
veranlaßt Moleküle, die physikalischen Gesetzmäßigkeiten unterliegen, strukturelle Stabilität und getreue Reproduktion anzustreben? «Die Natur»?

Die früheste Entwicklung, die Übergangsstufe von sogenannten primitiven Lebewesen (Bakterien) zu Eobioten (Biomoleküle), bleibt - da sie auch morphologisch unüberbrückbar zu sein scheint - das «dunkle Zeitalter» der Evolution, wie E.Broda diese Stufe nennt.

\section{Spezielle Problematik der genetischen Informationstheorie}

Das Erregende an der DNS-Basenanordnung ist für die Genetiker die «schriftartige Erbsubstanz»(C.Bresch). Dabei muß aber betont werden, daß die übertragene Gen-Information in das Protein durch die Boten-RNA keinerlei Materie («physical material»: M. Strickberger) enthält. Da dieser Informationsbegriff also nicht mehr physikalisch definierbar ist, müssen unspezifische Hypothesen gebildet werden, die den genetisch-biochemischen Prozeß «anthropomorphisieren», und zwar mit Hilfe der technologischen Analogie von Code und Decodierung (S.61 ff.). Es findet so nicht nur eine problematische Gleichsetzung von Information, Symbol, Nachricht und Anlage statt, sondern der menschliche Bewußtseinsprozeß wird seinerseits - als stillschweigendes Modell - auf «Programmierung» reduziert. Daß «Leben» in der Schlüsselsubstanz DNS «abrufbereit» gegeben ist, erscheint dann äußerlich als eine logische Konsequenz, die aber außer acht läßt, daß Zellen nicht in der selben Weise «Entscheidungen» über Zustandsänderungen fällen wie Computer-Programme. Im biologischen Lebensvorgang ist die quantitative Bestimmung der «Information» nicht ablösbar von ihrem Inhalt. Nimmt man hinzu, daß der Phänotyp eines Organismus nicht bloß eine Ansammlung aller Proteine darstellt, die den Genen des Genotyps entsprechen, dann läßt das «Kopieren» eines vorgegebenen Matrizenmusters eine wesentliche Frage ungelöst: Was befähigt im Grunde die DNA zu «Speicherung, Transport» und «Interpretation» im Sinne einer «alles übersteigenden hierarchischen Kontrolle, die die Handlungen des Organismus bestimmt» (Zit. H.H.Pattee, S.75)?

Solange diese Frage nicht beantwortet ist, dürfen auch die bisher unbekannten genetischen Teilprozesse nicht als ausreichender Beweis für eine «Evolution» durch «natürliche» Mikro-«Auslese» in Anspruch genommen werden. Und dahinter stellt sich zusätzlich das Problem des «Wachs- 
tums» einer Kopie innerhalb von «Grenzbedingungen», deren Erzeugung eben nicht mehr physikalisch oder chemisch spezifiziert werden kann. Wird jedoch ein System zugestanden - oder vorausgesetzt -, in welchem die «entstandenen informativen Konfigurationen (Folgen usw.) der Moleküle einen Sinn bilden» (Zit. A. Locker, S. 79), so bleibt an C.F.v. Weizsäcker zu erinnern, der schon 1971 hervorhob, daß der Begriff der Information nicht nur stets die «natürliche Sprache» schon voraussetzt, sondern auch ein «immateriell-materielles Etwas», welches weder «Ding» noch «Bewußtseinsinhalt» ist. Für D.Wyss (S.82f., $103 \mathrm{ff}$.) sollte daher der Begriff der «Form» mit in die Diskussion einbezogen werden, weil die informationstheoretischen Kausalerklärungen der biomolekular-organischen Prozesse aus epistemologischer Sicht sonst ein «typisches faute de mieux» bleiben.

\section{Die Vieldeutigkeit des Evolutionsbegriffs}

Was hinsichtlich des theoriefundierenden Begriffs der Evolution überrascht, ist sowohl sein vieldeutiger Gebrauch wie seine damit verbundene Verwechslung zwischen Bedingung und Ursache. Sieht man außerdem von der stillschweigenden, aber an sich unbewiesenen Voraussetzung ab, das Komplexe und Komplizierte habe sich stets aus dem Einfachen entwickelt (cf. S.157), so reicht das Evolutionsverständnis der Biologie von der global (vorwärts) gerichteten Artenentfaltung bis hin zur einfachen Größendifferenzierung im Zusammenhang mit (räumlicher) Umweltanpassung. Mutation, Selektion, Adaptation und Isolation können aber weder die «Stammbaum»-Frage im Sinne einer «Speziation» (A.Remane) zufriedenstellend lösen, noch eine wirkliche Antwort bezüglich der Neogenese von Organen, Phylen und Arten geben, die deshalb auch durch den bloßen Begriff der Epigenese «verharmlost» wird (S. 154f.).

Die Schwierigkeit liegt keineswegs bei den fossilen «Lücken» allein, wo Neuentdeckungen eher zu weiteren notwendigen Unterteilungen führen als zum Schließen dieser «Lücken». Die phylogenetische Systematisation selbst beruht u. a. auf «Intuition», die außer der Berücksichtigung von tabellarisch festgelegten Merkmalen dem Gesamteindruck eines Lebewesens unterliegt. Das heißt, die unverwechselbaren Eigenschaften werden zwar wahrgenommen, gehen aber gleichzeitig in ein bestimmtes perzeptives Apriori auf.

Auf dieser Zweideutigkeit beruht auch das sogenannte biogenetische Grundgesetz von E.Haeckel: Ähnlichkeiten der Ontogenie oder Keimesge- 
schichte beweisen nur dann phylogenetische Zusammenhänge, wenn der Glaube an die Abstammungsverwandtschaft vorausgeht (cf. S. 163). Und in dieser Hinsicht besitzt nicht nur die gleichmäßige Entwicklung als Umbildung der Arten einen fiktiven Charakter, sondern der Typen-Begriff selbst muß umstritten bleiben. Die Annahme der Orthogenesis («Gerade-Gerichtetheit») befindet sich auch im Widerspruch zu der Tatsache, daß die meisten bekannten Mutationen nicht in der von der Evolution bevorzugten Richtung liegen. Eine konsequent zu Ende gedachte Evolution als Optimierung von Spezialisierung führt geradewegs zu einem Paradox: Überspezialisierung provoziert Untergang in einer Population oder sogar deren Verschwinden insgesamt. Dies bedeutet, daß die «letztlich maximal geglückte Anpassung» (S.119), als Gipfel der «kreativ» gedachten Selektion, das selbsterzeugte Verhängnis der Evolution ist, obwohl ja gerade diese optimierte Anpassung theoretisch vom (Neo-)Darwinismus gefordert ist.

Ist mithin der Artenbegriff durch taxonomische Schwierigkeiten belastet, so erscheint andererseits das Anpassungsmodell - vornehmlich als «fitness» - wenig zu einer kausalen Erklärung in zeitlicher wie räumlicher Hinsicht beizutragen. Denn ein Überleben der «Geeignetsten» ist weitgehend tautologisch, weil die Frage nach dem Zweck der «Geeignetheit» immer schon den «Geeignetsten» voraussetzt. Und es gibt Fälle von ungewöhnlichen Strukturen bei Pflanzen und Tieren in außerordentlichen Lebenssituationen, die für die Fortpflanzung höchst ungünstig sind, wie zum Beispiel bei der Fischart der Kladozeren, wo die Jungfischschwärme zur Nahrung dienen.

Aber selbst wenn das Fortpflanzungskriterium der ursprünglichen Darwinschen «Lebenskampftheorie» heute zugunsten der genetischen Anpassung in den Hintergrund tritt, so vermag «fitness» immer nur a posteriori festgestellt werden. Und damit fallen nicht nur bedenkenlos «zwischen fünfzig und viertausend Millionen von ausgestorbenen Arten» einem «Konkurrenzkampf» von «frühkapitalistischer» Manier anheim (S.120, 135), sondern solcher Darwinismus erweist sich wiederum als «latenter Lamarkkismus» (Illies, zit. S. 137), da die Auslese am Phänotyp stattfindet.

Dies veranlaßt zu neuerlichen Hypothesen wie jener von «neutralen Genen» oder einem «genetischen Polymorphismus», bzw. es wird die Genvorstellung ganz aufgegeben, um rein «stochastische» Wahrscheinlichkeiten bei der Auslese am Werk zu sehen, also geographische, ökologische, existentielle, klimatische Zufälle u. a., um nur die «äußeren» zu nennen (cf. S. 97, 139 ff.). Anpassung selbst wird damit zu einem zufälligen Resultat von 
selektiven Faktoren, so daß der Beweis schwerfällt, ob nun überhaupt eine «Selektion» stattgefunden hat oder bloß eine «bessere Anpassung». Weiterhin muß logisch zugestanden werden, daß vorausgegangene Arten vor der «Anpassung» durchaus «überlebt» haben, falls die «neue» Art theoretischerweise immer «besseres Angepaßtsein» beansprucht.

Analoges gilt auch vom «systemtheoretischen» Begriff der Fulguration, den auch K.Lorenz vertritt. Entweder meint nämlich das «blitzartige» Auftreten von Neuschöpfungen oder neuen Eigenschaften einen von uns bisher noch nicht erkannten Kausalzusammenhang, oder es handelt sich um eine wissenschaftlich unzulässige creatio ex nihilo. Wie der Darwinismus also führt auch der «Fulgurationismus» zurück in den Reduktionismus oder den Präformationismus (cf. R. Löw, zit. S. 165).

Soll schließlich der Typenbegriff seine Geltung beibehalten, ohne daß er eine rein generalisierende Abstraktion bleibt, dann dürfte eine Begründungserforschung der Morphologie naheliegen, die nicht ohne einen immanenten Entfaltungstrieb der Organismen auskommt. Die Mannigfaltigkeit der Gestaltungsverhältnisse übertrifft in der Tat bei weitem, wie $R$. Woltereck schon früh erkannte (cf. S.145), die Größenordnung ehemaliger und heutiger Lebensbedingungen; sie ist nicht einfach «ökonomisch» im Sinne einer Energieverwertung (Stoffwechsel), wie letztere nunmehr von der Theorie der «non-darwinian evolution» als Kriterium angesetzt wird. Ein genauer bedachter Gestaltungsbegriff würde besagen, daß der Typus «eine nicht weiter zurückführbare Grundorganisation unterschiedlicher Lebewesen» darstellt, die sich dann ihre je spezifische «Um-welt» aktiv schaffen (S. 161, 165). Nur eine neolamarckistische Theorie wie die «Systemtheorie» kann solche «Anpassung», die an sich ein aktiver Prozeß bleibt, als im Gen gespeichertes «Erlernen» darstellen, um die eigentliche Antinomie in allem Lebendigen zu umgehen (vgl. Teil 5).

\section{Kybernetik und Organismus}

Die Grundauffassung von $D$. Wyss, daß alles lebendige Sein, bis hinein in die Gesetze der Entstehungs- oder Werdensvorgänge, von unaufhebbaren Gegensätzlichkeiten bestimmt bleibt, führt zu der prinzipiellen Frage, ob Organismus und technische (Regel-)Systeme überhaupt miteinander vergleichbar sind.

Die Diskussion darüber ist so lebhaft wie einst die Kontroverse zwischen 
Anhängern des Mechanismus und des Vitalismus. Ohne letzterem das Wort zu reden, besteht zunächst ein wesentlich methodischer Unterschied darin, daß der Biologe Lebensvorgänge beschreibt und der Ingenieur Maschinensysteme gedanklich oder technisch-industriell konstruiert, was Steinbuch übersehen möchte (cf. S. 172 ff.). Daß in heuristischer Hinsicht das kybernetische Modell seit N.Wiener (1947/48) zur Erforschung organischer und neurophysiologischer Vorgänge beigetragen hat, ist anzuerkennen. Aber ist damit auch das schon früher bekannte Prinzip der «Homöostase» und der linearen «Erregungs»-Symmetrie von «Reiz» und «Reaktion» eine wirkliche Gesetzmäßigkeit, die durch einen «Sollwert» festgelegt ist?

Bereits die Embryoentwicklung ist nur denkbar, indem ein bestehendes Gleichgewicht permanent aufgehoben wird. Und in ideengeschichtlicher Sicht ist die Gleichgewichtsvorstellung ein Produkt der Naturphilosophie des 19. Jahrhunderts. Nicht nur die Psychoanalyse wurde davon beeinflußt, sofern sie «seelisches Gleichgewicht» zu einem erstrebenswerten Ideal in der öffentlichen Meinung werden ließ, sondern auch die Innere Medizin und Pathophysiologie hat bis heute die «Norm»-Problematik zu keinem abgeklärten Abschluß führen können. Homöostase, so ist zu präzisieren, besteht eigentlich nur nach Stillstand von Stoffwechsel, d.h. nach dem Sterben eines Lebewesens. Bis zum «Tod» aber bleibt leibhaft-organisch gegebenes Menschsein vom Möglichen bestimmt oder vom intentionalen Welt- und Selbstbezug, welche erst im Nachhinein als «geregelte» und fest umrissene Intentionen erscheinen.

Bestimmt der Übergang vom Gleichgewicht zum Ungleichgewicht die Lebensprozesse (Katchalsky u. a., Zit. S.177), dann sind Organe mehr als vertauschbare «Größen» in unterschiedlichen Regelkreisen; es sind «Latenzzeiten» als Lebens-«Äußerungen» zu berücksichtigen, nach denen Wirkungen erst unter bestimmten Bedingungen in Erscheinung treten. Kybernetisch soll solche «Totzeit» mathematisch von den Zellen «verrechnet» werden. Wie jedoch ist diese proportionale, integrale oder differentiale (Zeit-)Systematik verstehbar ohne ein vorausliegendes «Gedächtnis» im Organischen selbst (cf. S.179f.)? D. Wyss beruft sich an anderer Stelle auf H.Bergson, wo dieser Gedächtnis-Begriff ( mémoire) zentral ist, um «Geist»Bewußtsein von «Materie» zu scheiden (cf. S. 225 und R. Kühn). Aber auch ohne diesen lebensphilosophischen Rekurs muß zugestanden werden, daß «organische Regelkreise» selbst der zeitlichen Wandlung durch Wachstum unterliegen. Uexküll, der alle Funktionsabläufe für physikalisch erklärbar hielt, sah nichtsdestoweniger in den zugrundeliegenden «Bauplänen» noch 
«ewige Schöpfungen», womit das Problem eines organischen Gedächtnisses diesseits wie jenseits der «Entwicklung» als Problem gestellt blieb.

Die Hypothese eines «Fließgleichgewichtes» umgeht nicht nur diese Schwierigkeiten von (Regel-)Sollwert und individuell-organischer Zeitlichkeit wie Befindlichkeit, sondern im Grunde wird auch die theoretische Grundforderung eines streng linear-kausalen Informations-Regelflusses durch das Modell der «Vermaschung» aufgegeben. Denn die biologische Kybernetik setzt in einer "probabilistic approach» (Keidel, Zit. S.183f.) «zeitliche und örtliche Summationen» voraus, um der Verzweigung und Verteilung von Milliarden zellularen Prozessen gerecht zu werden, die in gleichzeitig-fortlaufender Überlagerung untereinander «Impulse» weitergeben. Eine solche «Vermaschung der Regelkreise», um gegenüber der kybernetischen Konstanzanforderung getreu zu bleiben, umgeht dabei die zusätzliche Frage der an sich von ihr geforderten linearen Gleichzeitigkeit durch den erweiterten Begriff einer «zirkulären Kausalität». Deren wissenschaftstheoretisches Apriori wiederum ist dann, daß Phänomene der Wechselwirkung «Rückkoppelungsmodellen» entsprechen, die letztlich kosmisch fundiert sind: «Der gesamte Kosmos kann als ein hierarchisch organisiertes System betrachtet werden», wodurch alle «Netzwerkmodelle» zu «abstrahierten Abbildern» einer Vorstellung werden, welche selbst wiederum nur ihre «funktionelle Organisations(abkünftigkeit)» zum Ausdruck bringt (F.M.Wuketits, Zit. S. 185).

Diese wohl kaum anders denn «metaphysisch» zu nennende Ausweitung des Kybernetikmodells durch ihre Vertreter selbst bliebe wiederum ein philosophisch-epistemologisches Binnenproblem, wenn die «Human»-Wissenschaften es nicht schon praktisch umgesetzt hätten in institutionelle «Lern»-Strategien. So ist Lernen, wie G. Walters programmierte «Schildkröte», vermehrt zur Informationsspeicherung und -vermittlung geworden, ohne da $\beta$ immer bedacht wird, daß beim wirklichen Lernen neue Informationen gebildet werden und kein prädeterminiertes Programm nur noch präzisiert bzw. abgerufen wird.

Ohne in eine grundsätzliche Technikfeindlichkeit zu verfallen, ist mit H.Jonas (cf. S.185f.) der eigentümliche zeitgenössische Bewußtseinszustand einer «metaphorischen» Zweideutigkeit nicht zu verkennen. Die Artefakte nehmen menschliche Funktionen an, während die Menschen zu technisch-schematisierten Funktionsträgern werden. Daß die Unterscheidung von Mensch und Maschine in dieser Verkehrung oder Doppelsinnigkeit dahinzielt, dem Befehlen oder Zweck-Dienen Vorrang einzuräumen, ist 
spätestens seit LaMettries l'homme machine ein Zug der Moderne, die Autonomie nur als Selbstschöpfung noch verstehen kann. Für diese Diskussion darf ergänzend auf die philosophie-technischen sowie -medizinischen Arbeiten u. a. von A. Baruzzi und C.Bruaire verwiesen sein, da hierbei auch das Verhältnis von staatlicher Macht und deren Verfügung über wissenschaftliche Standards zur Sprache kommt.

\section{Zusammenfassung der Evolutionskritik}

Für D. Wyss liegt die «Hybris und Unredlichkeit der modernen Biologie», wie das letzte VI. Kap. lautet (S.217-237), in ihrer tendenziellen «nihilistischen» Reduktion lebendiger Ordnungsgefüge. Die Gefahren, die sich daraus konkret schon jetzt ergeben, erscheinen vor allem als «Vergessen» der sekundären Sinnesqualitäten (Farbe, Ton, usw.) zugunsten mechanischer Abstrakta wie Kraft und Bewegung z. B. Dahinter verbirgt sich der Verlust an weiteren biologischen Grundeinsichten, die sich aus den detailliert-exegetischen Beobachtungen seitens Wyss' über biochemische und informationskybernetische Reduktionsmodelle in den vorangegangenen fünf Kapiteln ergeben und wie folgt zusammenfaßbar sind:

1) Elektronenverschiebungen sollen den Urrhythmus von Assimilation und Dissimilation ersetzen.

2) Die «Sinnfülle» der Lebensvorgänge erscheint wegstilisiert durch evolutionäre «Zufälle» oder «Wahrscheinlichkeiten», die vorgeben, alle «Welträtsel» zu lösen.

3) Erkenntnistheoretische Fragen verändern sich in Probleme der «richtigen» Information zwecks «Überleben», welches ein «vorbewußter, ratiomorpher Verrechnungsapparat» - nach Maßgabe eines «eingebauten hypothetischen Realismus» - schon immer vorentscheidend intendiert hat.

4) Aber nicht nur Intentionalität geht in «Verrechnen» auf, sondern auch die Willensfreiheit ist bloß die letzte Stufe von Mutationen, die sich auf dem Weg von «trial and error» als Motor der Evolutionsspirale erweisen (Riedl, Zit. S.221 f.).

5) Bewußtsein und Intentionalität fallen «evolutionär» mit der «Außenwelt» zusammen. Damit ist der Natur kategorial unterstellt, was anschlieBend genetisch als Denkkategorien aus ihr abgeleitet wird. Anstatt einer letzten, reflexiv unüberbrückbaren «Kluft» (S.223) zwischen Mensch und 
Welt geht anthropologisch begründetes Kausalitätsdenken in einen Zirkelschluß über: kausales Denken ist weltangemessen, weil es sich in der Welt behauptet (cf. Löw, Zit. S. 223 f.).

6) Sofern systemtheoretisch die Wechselwirkung zwischen Organismus und Umwelt als Optimierungsvorgang auftritt, fehlen (Wert-)Kriterien für ein «besseres» Überleben: entweder ist dieses tautologisch oder eine «Willkür-Norm». Es sei denn, der «technologische Homo faber» sei die Norm schlechthin, die sich (bis jetzt!) bestens zum Überleben gegenüber den Tieren erwiesen hat (S.229f.).

7) «Teleonomie» (Pittendrigh, Monod, Zit. S.230f.), die sich von jeder «Teleologie» distanziert, ist reine $A l s-O b$-Betrachtung von zweckmäßig erscheinenden Funktionen der Arterhaltung. Damit verschiebt sich das Finalitätsproblem auf die «mystifiziert»-programmierte Erbmasse und/ oder die Selektion, die «blind» und «zufällig» die Arterhaltung - als Zweck! bestimmen.

8) Da Evolutionshypothesen auf Plausibilitätsschlüsse und Analogien rekurrieren, die weder falsifizierbar noch verifizierbar sind, ist der biologische «Wahrheitsbegriff» nomothetisch-logisch begründet, dessen «prius» in den molekularbiologischen Valenzen ruht. Wie einst der «Wille Gottes» oder der «absolute Geist» herrscht jetzt der «materialistische Monismus» im Gewand von Information und Erbzufällen als das neue «Absolute» (S. 233 ff.).

\section{Entwurf einer antinomischen Lebens- und Evolutionsstruktur}

Wenn jedoch überhaupt etwas als «absolut» - oder besser: als «unendlich»in praktischer Beschreibungshinsicht für biologische Charakteristika bezeichnet werden darf, dann sind es «die überwiegend diskontinuierlichen Vorgänge bei aller gleichzeitigen Kontinuität der Prozesse» (S. 197 ff.). Im Anschluß an die von Woltereck herausgearbeiteten phänomenalistischen «Wesensmerkmale» der Selektivität, Reaktivität, Impulsivität, Spontaneität, Autogenie, Autergie und Autotelie der Organismen, welche sich ihre Umwelt stets in antagonistischer Auseinandersetzung gestalten, ergibt sich die Einheit der Natur in ihrer Vielheit, ohne dieses Verhältnis logisch auflösen zu können.

Außer der bereits erwähnten Rhythmik der kata- und anabolen Prozesse im Stoffwechselbereich z. B. unterliegen auch die Wahrnehmungsvollzüge 
den «Reizen» der Außenwelt dergestalt, daß sie dieselben als «Erscheinungen» gleichzeitig mitgestalten. Zwar ist jede Zelle wie eine «Monade» zu betrachten, und dennoch kann ihre Ausrichtung auf den organismischen Gesamtverband keineswegs übersehen werden. Schon aus diesen Beispielen folgt, daß kausal-mechanistische Erklärungen nur Teilvorgänge ergreifen, welche der «Interaktionalität» des Ganzen unterzuordnen sind. Aus diesem Grunde besitzt auch jedes organische Gebilde «mehrere spezifische Bedeutungen», die weder finalistisch noch dialektisch hinweginterpretiert werden können.

Eine Wissenschaftstheorie, die Aristoteles, Kant, Hegel und deren neueren Variationen folgt, kann deshalb nicht nachvollziehen, daß «die Lebensvorgänge sich in einer dem menschlichen Erkenntnisvermögen entziehenden Weise ereignen». Im Sinne einer solchen grundsätzlichen Aporie oder Antinomik sind «die Lebensprozesse als fundamental irrational zu bezeichnen» (S.201).

Was folgt daraus für einen «antinomischen Entwurf» der Evolutionsgeschichte? Prinzipiell:

«Es gibt nicht (verschiedene) Leben, wohl (aber) mannigfache Lebensvorgänge und Arten, die alle nach den gleichen fundamentalen Prinzipien «leben»» (S. 203).

Aus der Anwendung dieses Grundsatzes einer lebendigen Ordnung folgt insbesondere dann, daß Evolution sowohl Neo-/Epigenese wie bloße Veränderung vorhandener Merkmale ist. Das Leben «löst» den mono-/polyhyletischen Gegensatz des einen Lebens, indem neue Arten entstehen, so wie vorhandene sich zugleich evolutiv verändern. Die Mutationen sind dabei als «gerichtet» zu interpretieren, was den insgesamt (genetisch) umgestalteten Organismus betrifft. Mit Blick auf andere Tierarten - oder gar in bezug auf die «Gesamtevolution» - erscheinen mutative Änderungen als «ungerichtet». So gelangte die Entwicklung der Equiden bis zu den heutigen Pferden, die der Ammoniten beispielsweise aber führte zu einem Stillstand. Soll der rezessiv vererbte Phänotyp nicht einfach eine «maskierte» oder «verschwundene» (Gen-)Information darstellen, so vermag die Gleichzeitigkeit von «Akt und Vollzug» nur noch in einer gewissen Angemessenheit durch den «Potenz»-Begriff erfaßt werden.

Im Rahmen bisheriger biologischer Beobachtungen löst die Annahme einer (potentiell) «immanenten Richtung» weder das Problem der Anpassung durch geographische Isolation noch die Frage der taxonomischen Ordnung, aber «Potenz» erlaubt konzeptuell eine bescheidene Annäherung 
an das «Unbekannte» der lebendig-antinomischen Prozesse (S.205, cf. $212 \mathrm{f}$.$) . «Potenz», anders gesprochen, läßt eine Anpassung an spätere$ Umstände zu, ohne daß die «Kontinuität» präadoptionistisch «aktualisiert» wird, denn jedes Lebewesen ist durchgehend alloplastisch wie alloxen zu sehen. D.h. seine erstaunlich umweltangepaßte Plastizität vollzieht sich bei simultan weitgehend festgelegter genetischer Merkmalstabilität, die dem Umweltdruck als entzogen auftritt. «Veränderung» und «Ruhe» sind andere Beschreibungsmöglichkeiten der sich überall einstellenden Lebensantinomik, die «Mangel» erzeugt, um ihn vorübergehend zu kompensieren.

An der dabei «je-einmaligen» Leistung durch ein Lebewesen geht der «geniale» Kunstgriff der darwinistischen Verbindung von Selektion und Anpassung vorbei. Er verschweigt, daß solcher Umweltbezug taxonomisch stets individuell mitbedingt ist, so daß Selektion weniger richtungsdeterminierend ist denn stimulierend oder «feilend». Denn «ein Lebewesen ohne Umweltbezug - Anpassung - existiert nicht» (S.209)! Deshalb muß Evolution sowohl als «(Sinn-)Richtung» wie als «(Spiel-)Zufall» gelten gelassen werden, da eine finalistische Sinnfestschreibung zu unbegründbaren Hypostasen von Sinn führt. Die Sinnkategorie liegt «jenseits» menschlicher Zugriffsmöglichkeiten.

Entgegen dem naiv-herkömmlichen Zeitbegriff der Evolution als eines konstatierbaren Nacheinanders, worin ein unidimensionales (Sinn-)Ziel implizit vorgegeben ist, kann Zeit - nach quantentheoretischer Sicht - auch als zusätzliche, vierte Raumdimension gesehen werden. Differenzierung und Komplexität der Lebewesen wäre, so gesehen, die gestufte und artenentsprechende «Durchgestaltung» des Raumes und:

«Evolution (erschiene) bei einer relativ gleichbleibenden «Masse` von Lebewesen, Arten, (als) ein jeweils durch Bewegung (Veränderung) sich vollziehender Ausdehnungs- und Schrumpfungsprozeß» (S.211).

Dadurch wird der Zeitpfeil ebenfalls umkehrbar wie der Begriff der «Höherentwicklung» relativierbar; Anfang und Ende, Mensch und Einzeller, sind ineinander verschränkt, und die Evolution bekäme einen antinomisch-zyklischen Charakter.

Ob damit das bisher linear-«progressiv» gedachte biogenetische Grundgesetz in re-volutionärer Weise erschlossen wird, unterliegt der Frage nach dem Wesen des Zyklischen, welches eine seit altersher konkurrierende Vorstellung gegenüber jener der linearen Dialektik ist. Zwar hebt die 
zyklische Verschränkung von Anfang und Ende die «Hybris» der Höherentwicklung als Legitimierung (technisch-)humaner Selbstbehauptung z.T. auf, aber die Überlegung führt zur problematischen Annahme einer «zeitlosen Ordnung» im Sinne einer «hierarchischen Gedächtnisordnung», deren Wertung ihrerseits «intuitionistisch» ist, wenn auch unter Verweis auf den mathematischen Intuitionismus (cf. S.210). Immerhin umschließt diese «Intuition», sieht man von jeder speziellen Lebenswertung ab, die transzendentale Grundantinomik von Raum und Zeit. Denn wie immer auch deren Ineinander oder Auseinanderhervorgehen gedacht wird - die raum-zeitliche Herausforderung bleibt das nicht einholbare Apriori alles Lebendigen, sofern dieses identisch ist mit Strukturierungs- oder Gestaltungsaufgaben überhaupt.

Anstelle eines bereits sprachlich verschleiernden «Es», welches im mechanisch-finalistisch biologischen Denken oft die tatsächliche «Kreativität» der Evolution negiert, ist anzuerkennen, daß «Selbstdarstellung der Lebewesen über Umweltveränderung» stattfindet. Anstatt also abschwächend, reduktionistisch z.B. zu sagen: «Es wird Umwelt in Form von Nahrung aufgenommen», muß der Satz gewagt werden:

«Umwelt wird zu Lebewesen, Lebewesen zur Umwelt. (Dies ist) als die unendliche Variation beider zueinander, das zentrale Thema der Evolution überhaupt» (S.213).

So betrachtet, ist dann auch die «hierarchische Ordnung» der ineinander verschränkten Phyla, Genera, Familien usw., in ihrer räumlichen Verzeitlichung, keine statisch-fixierte Ordnung, sondern alle taxonomischen Zyklen werden «stets in Frage gestellt durch individuelle Abweichungen innerhalb von festgelegten Grenzen» (S.214f.). Die Alloplastizität bei der morphologisch-biochemischen Vererbung solcher «Sprünge» ist dabei allerdings noch einmal antinomisch in geschichtlicher Hinsicht zu differenzieren: überwiegt am Anfang «lamarckistische» Entwicklung, so wird gegen Ende hin die Evolution zunehmend «darwinistischer».

Diese tendenzielle «Vereinigung» von Lamarckismus und Darwinismus ist keine Synthese im versöhnenden Sinne, weil dies der antinomische Ansatz selbst nicht zuließe. Im Interesse des Lebens selbst ist vielmehr jene «tödliche» Evolutionserscheinung noch einmal in den Mittelpunkt zu rücken, die sich schon aus der Untersuchung des «Anpassungs»-Begriffs ergab: die Rigidität der ausdifferenzierten und festgelegten Formen besitzt zwar eine größere Stabilität als der lernfähig-plastische, undifferenzierte Beginn, aber dafür auch eine größere «Todesnähe»! 
Die erkenntnisleitende Anerkennung eines antinomischen und indeterministischen «Irrationalismus» im Lebensgeschehen beinhaltet also keineswegs Abdankung an "Wissenschaftlichkeit» oder an "Verantwortung». Eine solche Position veranlaßt im Gegenteil dazu, die Bedrohnis durch «Zerstörung» seitens nicht-alloplastischen Lernens für die Zukunft - aus dem Vergangenen - selbst «wiederzuentdecken». Was Biologie und alle Wissenschaften, die eine Evolutionstheorie voraussetzen, (noch) zu leisten haben, ist damit abgesteckt.

\section{Literatur}

Baruzzi, A., Mensch und Maschine. Das Denken «sub specie machinae», Fink, München 1973.

Bergson, H., L'évolution créatrice, Alcan, Paris 1907; dt.: Schöpferische Entwicklung, Diederichs, Jena 1912.

Broda, E., The evolution of the bioenergetic process, Pergamon Press, Oxford 1978.

Bruaire, C., Une éthique pour la médecine, Fayard, Paris 1978; dt.: Ethik für Mediziner. Von der ärztlichen Verantwortung zur moralischen Verpflichtung, Patmos, Düsseldorf 1980.

Eigen, M./Winkler, R., Das Spiel, Piper, München/Zürich 1978.

Illies, J., Schöpfung oder Evolution, Edition Interfrom, Zürich 1979.

Jonas, H., Organismus und Freiheit, Vandenhoeck \& Ruprecht, Göttingen 1973.

Keidel, W.D., Grenzen der Übertragbarkeit der Regelungslehre auf biologische Probleme, in: Die Naturwissenschaften 8 (1961) $271 \mathrm{ff}$.

Kühn, R., Zwischen Gedächtnis und Antizipation: der Augenblick. Philosophie und Psychologie der Zeit nach Alfred Adler in psychotherapeutischer Sicht, in: Petzold, H., Die Zeit in der Psychotherapie, Junfermann, Paderborn 1988.

Locker, A. (Hrsg.), Biogenesis, Evolution, Homeostasis, Springer, Berlin/Heidelberg/New York 1973.

Löw, Leben aus dem Labor: Gentechnologie und Verantwortung. Biologie und Moral, München 1985.

(zusammen mit R.Spaemann), Die Frage Wozu?, Piper, München/Zürich 1981.

Lorenz, K., Die Rückseite des Spiegels. Versuch einer Naturgeschichte menschlichen Erkennens, Piper, München/Zürich 1973.

Monod, J., Le hasard et la nécessité. Essai sur la philosophie de la biologie moderne, Le Seuil, Paris 1970.

Pattee, H.H., Experimental approaches to the origin of life problem, in: Advances in enzymology 27 (1966).

Remane, A., Die Grundlagen des natürlichen Systems der vergleichenden Anatomie und der Phylogenetik, Akademische Verlagsgesellschaft, Leipzig, 2. Aufl. 1956.

Strickberger, M. W., Genetics, Macmillian, New York, 2. Aufl. 1968.

Weizsäcker, C. F. von, Einheit der Natur, Hanser, München 1971.

Woltereck, R., Grundzüge einer allgemeinen Biologie, Enke, Stuttgart, 2. Aufl. 1940.

Wuketits, F. M., Biologie und Kausalität, Parey, Berlin/Hamburg 1981. 


\section{Zusammenfassung}

Der Autor diskutiert die Schwierigkeiten bei der Erklärung der Entstehung der Lebewesen nach der Evolutionstheorie von Darwin. Seine Darstellung basiert hauptsächlich auf einer neueren Untersuchung zu diesem Thema von Dieter Wyss.

\section{Summary}

The author discusses the difficulties with the explanation of the origin of species according to Darwin's Theory of Evolution. His point of argument bases mainly on a new interpretation of this theme by Dieter Wyss.

\section{Dr. Rolf Kühn}

Widerholdstraße 14

D-7700 Singen/Htw. 